\title{
Tectonomagnetic study in the eastern part of Hokkaido, NE Japan: Discrepancy between observed and calculated results
}

\author{
Yasunori Nishida, Yasuhiro Sugisaki*, Kosuke Takahashi ${ }^{\dagger}$, Mitsuru Utsugi $^{\ddagger}$, and Hiromitsu Oshima ${ }^{\circledR}$ \\ Division of Earth and Planetary Sciences, Graduate School of Science, Hokkaido University, Sapporo 060-0810, Japan
}

(Received February 23, 2004; Revised September 1, 2004; Accepted October 8, 2004)

\begin{abstract}
Continuous and repeated geomagnetic observations have been performed at 8 stations in the eastern part of Hokkaido, NE Japan, to confirm a detailed picture of geomagnetic secular changes. The observation delineated anomalously large secular changes of about $1 \mathrm{nT} /$ year that have lasted at least for about 3 to 30 years (depending on the period of observations) at 3 stations situated in the remarkable geomagnetic anomaly region. Contributions from the earth's core or ionospheric origin are ruled out as source mechanisms because of the local distribution of the anomalous stations. Heat-triggered volcanomagnetic effect cannot be the origin of such secular changes because the stations are quite far from the volcanoes. Instead, we propose the changes originate from stressinduced tectonomagnetic effect (piezomagnetism). We performed piezomagnetic modeling under the condition that observed regional tectonic stress has been applied to the highly magnetized rock bodies inferred from the analysis of the observed geomagnetic anomalies. The modeling explained well the secular changes by assuming the stress sensitivity of the order of $10^{-2} \mathrm{MPa}^{-1}$, which is one order larger than the ordinarily used value.
\end{abstract}

Key words: Geomagnetic secular change, tectonomagnetism, stress sensitivity.

\section{Introduction}

Stress-induced tectonomagnetism (piezomagnetism) has been studied by many researchers because it can give us information on stress changes within the crust independently of mechanical means. Analytical and numerical modelings have progressed considerably since the 1960's to provide us powerful tools for calculating the piezomagnetic fields for various types of pressure source (e.g., Stacey, 1964; Yukutake and Tachinaka, 1967; Sasai, 1980; Zlotnicki and Cornet, 1986; Oshiman, 1990; Sasai, 1991a, b; Sasai, 1994; Utsugi, 2000; Utsugi et al., 2000; Sasai, 2001).

Laboratory experiments have revealed that stress sensitivity of piezomagnetism is the order of $10^{-3} \mathrm{MPa}^{-1}$ for stiff rocks such as basalts and andesites (e.g., Kapitsa, 1955; Nagata and Kinoshita, 1967). On the other hand, Hamano (1983) found that some porous rocks such as tuff have the stress sensitivity of about $10^{-2} \mathrm{MPa}^{-1}$ and concluded that stress sensitivity increases with porosity of rocks. Stress sensitivity also depends on titanium content in titanomagnetites (Stacey and Johnston, 1972) and magnetic grain size (Kean et al., 1976).

Many field observations associated with seismic and

\footnotetext{
* Now at: NEC Software Co. Ltd.

${ }^{\dagger}$ Now at: Research Center for Prediction of Earthquakes and Volcanic Eruptions, Graduate School of Science, Tohoku University.

${ }^{\ddagger}$ Now at: Institute for Geothermal Sciences, Graduate School of Science, Kyoto University.

ฯ Now at Institute of Seismology and Volcanology, Graduate School of Science, Hokkaido University.
}

Copy right(c) The Society of Geomagnetism and Earth, Planetary and Space Sciences (SGEPSS); The Seismological Society of Japan; The Volcanological Society of Japan; The Geodetic Society of Japan; The Japanese Society for Planetary Sciences; TERRAPUB. other tectonic activities have succeeded in detecting the piezomagnetic changes as in examples of the San Andreas fault system, USA (Johnston et al., 1985), the Landers earthquake, USA (Johnston et al., 1994), a Parkfield fault model, USA (Stuart et al., 1995), the Izu Peninsula earthquake, central Japan (Sasai and Ishikawa, 1997) and so on. In some cases, however, tectonic activity failed to generate expected magnetic changes (e.g., Sasai and Ishikawa, 1980).

The observation of geomagnetic changes during the filling of reservoir (the dam magnetic effect) is regarded as a large-scale control experiment. Magnetic changes were observed at Talbingo reservoir in Australia (Davis and Stacey, 1972), Charvak reservoir in ex-USSR (Abdullabekov et al., 1979), Miyun reservoir in China (Zhan, 1989), Nichinan reservoir in Japan (Oshiman et al., 1991a) and so on. The observed amplitudes tend to be much larger than those calculated when we assume a stress sensitivity of the order of $10^{-3} \mathrm{MPa}^{-1}$ based on the laboratory experiments.

The eastern part of Hokkaido, NE Japan, is situated in the southwestern end of the Kuril Arc and is a tectonically important place where a collision of the North American plate with the Eurasia plate took place in the late Cretaceous or Paleogene (Niida and Kato, 1986). In addition to such past tectonic activity, subduction of the present Pacific plate is transforming Hokkaido, resulting in a complex tectonic setting (Fig. 1) and occurrence of large earthquakes. Triangular surveys in this region have revealed dominant crustal contraction $\left(4 \times 10^{-7} /\right.$ year) with principal axis of NW-SE direction, being almost perpendicular to the trench axis as shown in Fig. 2 (Ishikawa and Hashimoto, 1999). In the present paper, we discuss the geomagnetic changes related with the tectonic stress field in this region. 


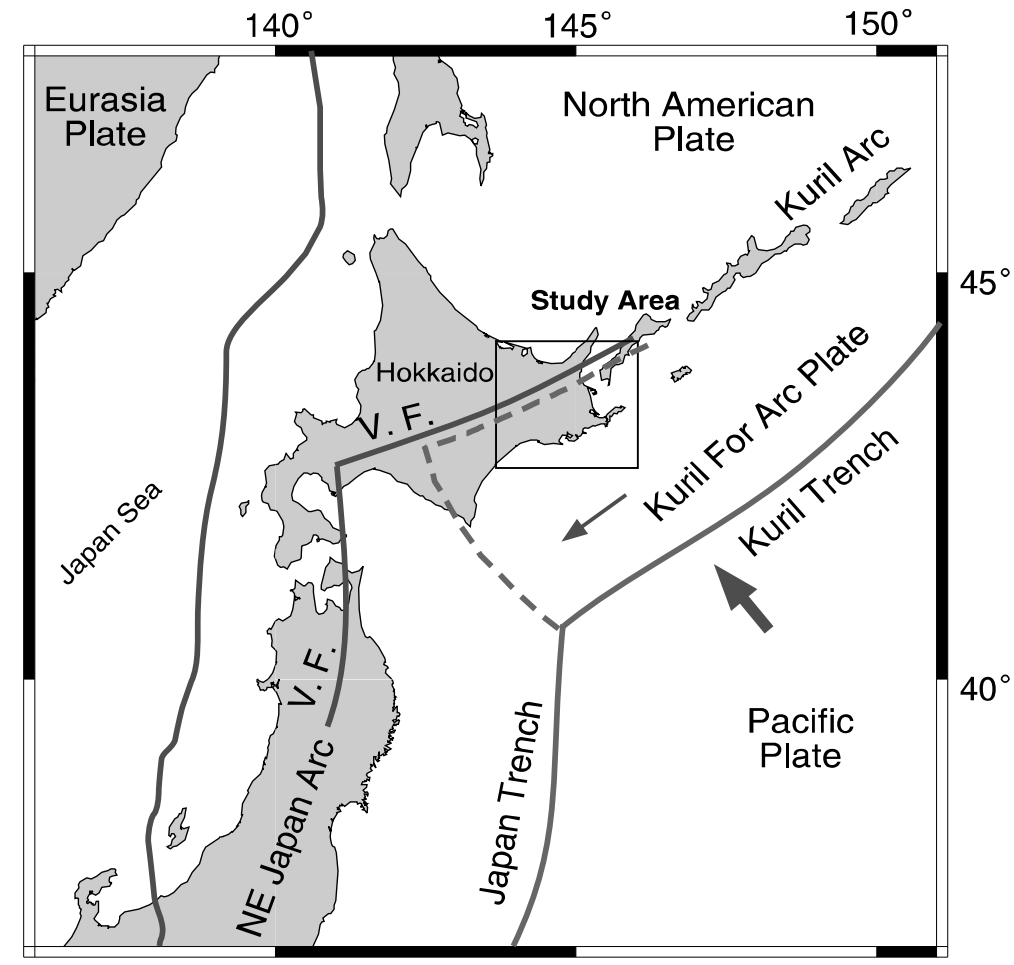

Fig. 1. Tectonic framework in and around Hokkaido, Japan. A thin arrow denotes the direction of the crustal movement caused the collision of the Kuril Arc against the NE Japan Arc. A bold arrow indicates the direction of the Pacific plate motion. VF indicates the volcanic front.

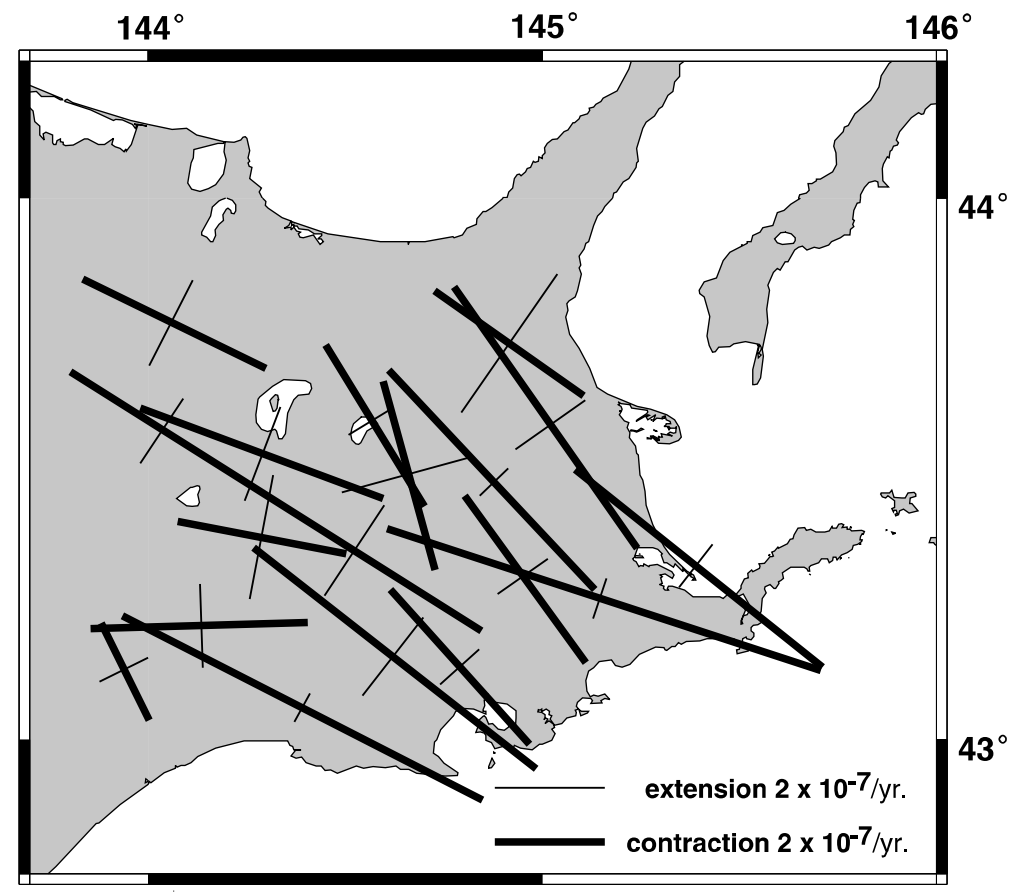

Fig. 2. Distribution of principal axes of strain rate. Bold and thin bars indicate axes of contraction and extension, respectively (modified from Ishikawa and Hashimoto, 1999).

\section{Repeated and Continuous Observations of the Geomagnetic Field}

Hokkaido University had performed irregularly repeated observations of the geomagnetic total force at AKS and SHI (Fig. 3) from 1972 to 1994 to detect the tectonomagnetic signals (Oshima et al., 1994). Memanbetsu Magnetic Observatory (Japan Meteorological Agency) also made re- peated observations at ATK and MID (Fig. 3) from 1971 to 1982 (Hasegawa et al., 1983). These observations had detected large secular changes at AKS and ATK. However, we had much difficulty in interpreting these data with only a few stations. We established additional 6 stations for continuous measurements at AKS, KKM, SHI, NIT, NIB and NMR during the period from August 1996 to November 


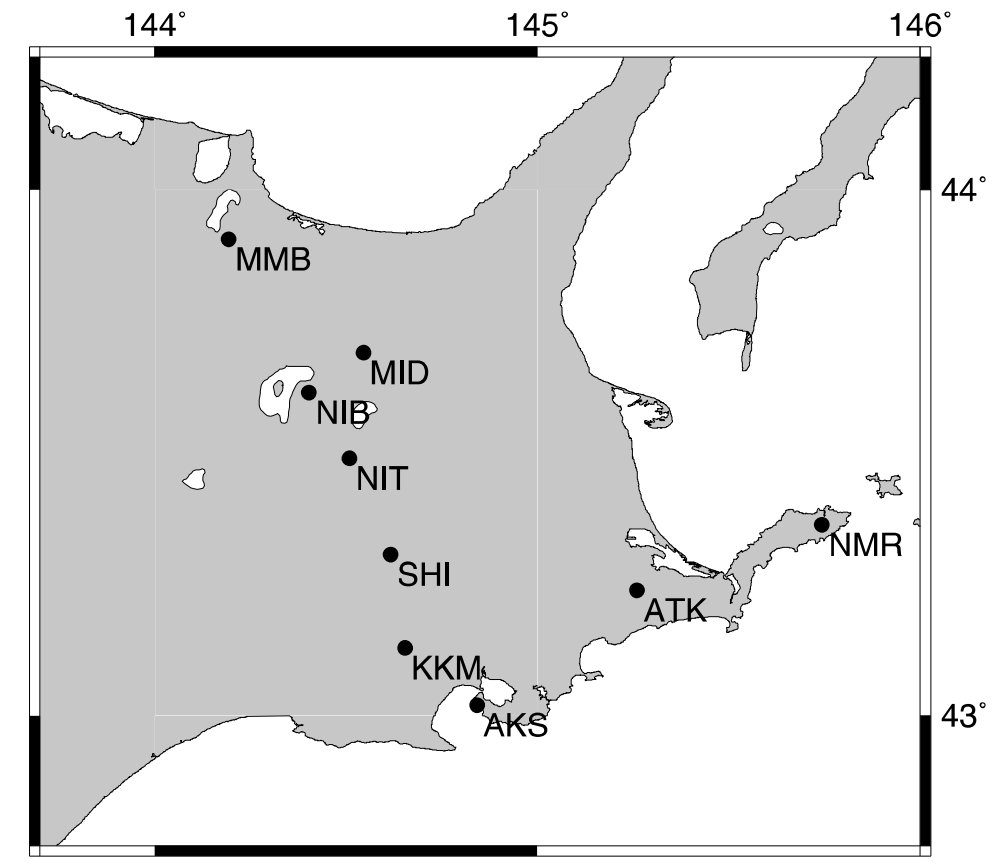

Fig. 3. Continuous and repeated geomagnetic stations are shown by solid circles. ATK and MID were established by Hasegawa et al. (1983).
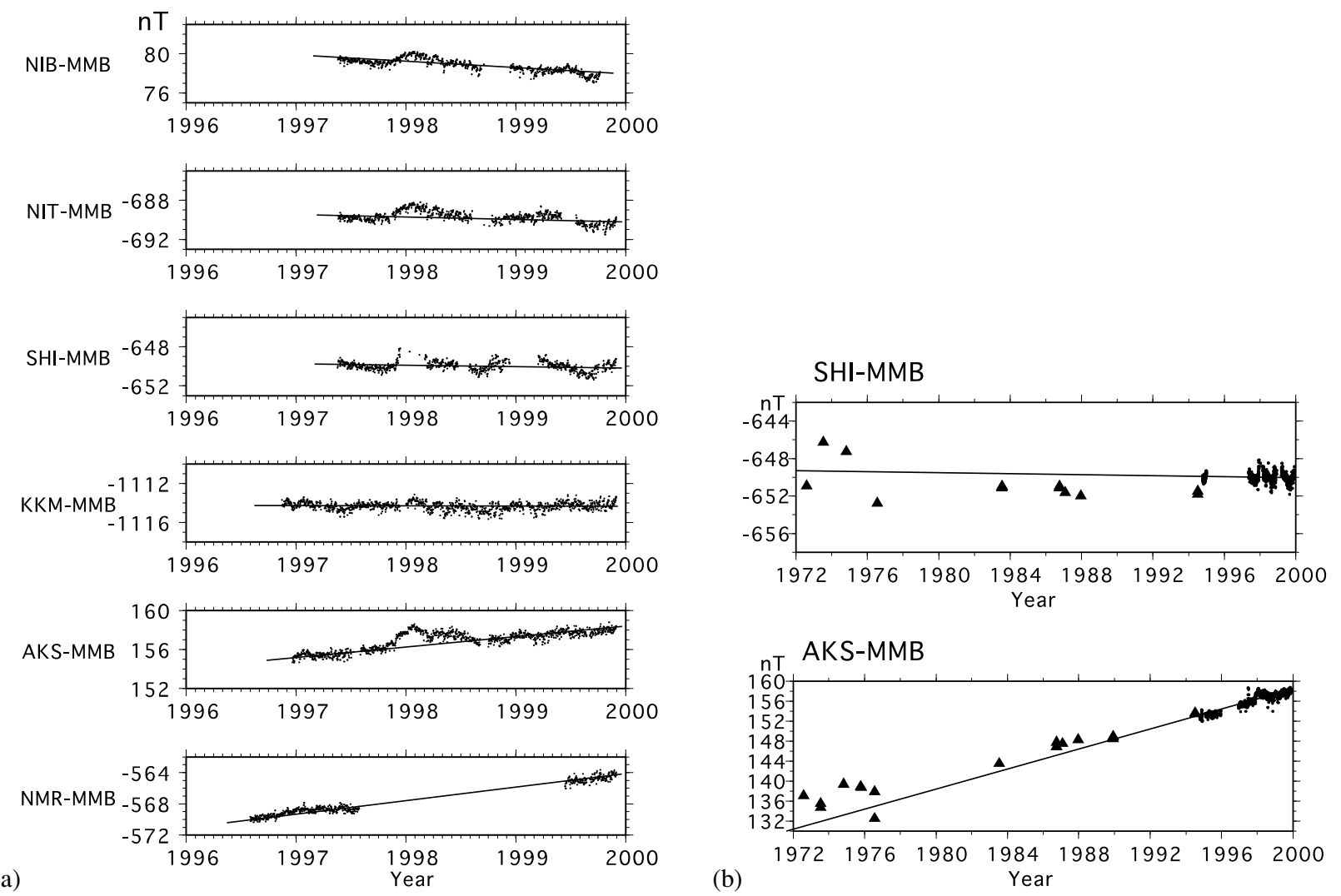

Fig. 4. (a) Whole-day mean simple differences of the total force intensity between each station and Memanbetsu Magnetic Observatory (MMB) during the period from August 1996 to November 1999. Linear least-squares regression lines are also shown. (b) Temporal changes of the total force intensity at AKS and SHI. The changes obtained by repeated measurements are shown by solid triangles, while whole-day mean simple differences shown in Fig. 4(a) are plotted by solid circles. Linear least-squares regression lines applied for the periods from August 1996 to November 1999 are extraporated to 1972 .

1999 to confirm a detailed picture of the secular change in this region (Fig. 3). The stations except NMR were aligned perpendicularly to the trench axis of the Pacific Ocean and in almost parallel to the principal axis of the tectonic con- traction. Total force of the geomagnetic field was measured at every 1 minute using proton precession magnetometers. The observed values at each station were subtracted from values at the reference station MMB (Memanbetsu Mag- 


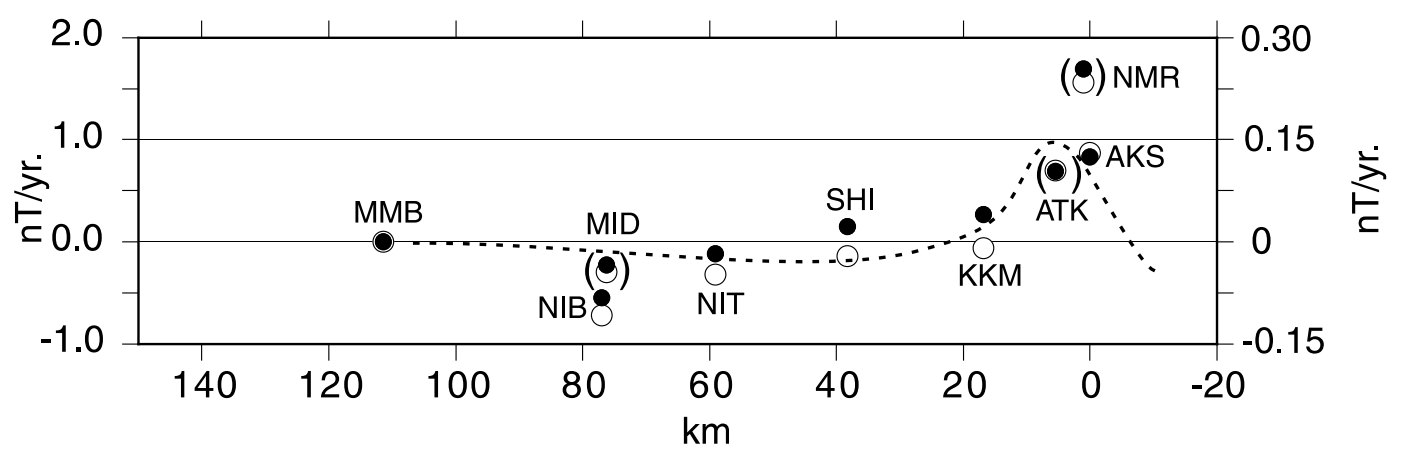

Fig. 5. Observed secular changes (open circles) and corrected ones for the orientation effect (solid circles) are plotted against the horizontal distance from AKS to MMB (L1 line in Fig. 6). Amplitudes are referred to the left-side ordinate. For convenience of comparison, the values at NMR, ATK and MID are also projected onto the measurement line. Calculated piezomagnetic field is shown by a dashed curve (right-side ordinate). Calculated maximum amplitude is compared with the corrected value at AKS. Both the stress sensitivity of remanence and susceptibility are assumed to be $2 \times 10^{-3} \mathrm{MPa}^{-1}$ for the calculation.

Table 1. Observed secular changes and corrected ones for the orientation effect. The reference data for reduction is obtained at Memanbetsu Magnetic Observatory (MMB).

\begin{tabular}{|c|c|c|c|c|c|c|}
\hline \multirow[t]{2}{*}{ Station name } & \multirow[t]{2}{*}{ Abb. code } & \multicolumn{2}{|c|}{ Location } & \multirow{2}{*}{$\begin{array}{c}\text { Secular change } \\
\text { nT/year }\end{array}$} & \multirow{2}{*}{$\begin{array}{c}\mathrm{SD} \\
\mathrm{nT} / \text { year }\end{array}$} & \multirow{2}{*}{$\begin{array}{c}\text { Corrected secular change } \\
\text { nT/year }\end{array}$} \\
\hline & & lat. $\mathrm{N}$ & long. E & & & \\
\hline Akkeshi & $\mathrm{AKS}$ & $43^{\circ} 10.2^{\prime}$ & $144^{\circ} 50.5^{\prime}$ & 0.87 & 0.03 & 0.83 \\
\hline Kitakata-musari & KKM & $43^{\circ} 07.8^{\prime}$ & $144^{\circ} 50.5^{\prime}$ & -0.06 & 0.02 & 0.27 \\
\hline Shibecha & SHI & $43^{\circ} 18.5^{\prime}$ & $144^{\circ} 37.0^{\prime}$ & -0.14 & 0.03 & 0.15 \\
\hline Nita & NIT & $43^{\circ} 29.5^{\prime}$ & $144^{\circ} 30.5^{\prime}$ & -0.32 & 0.03 & -0.12 \\
\hline Nibetsu & $\mathrm{NIB}$ & $43^{\circ} 37.0^{\prime}$ & $144^{\circ} 24.2^{\prime}$ & -0.72 & 0.02 & -0.55 \\
\hline Nemuro & NMR & $43^{\circ} 21.9^{\prime}$ & $145^{\circ} 44.6^{\prime}$ & 1.56 & 0.02 & 1.69 \\
\hline Attoko & ATK & $43^{\circ} 14.4^{\prime}$ & $145^{\circ} 15.6^{\prime}$ & 0.70 & - & 0.69 \\
\hline Midori & MID & $43^{\circ} 41.5^{\prime}$ & $144^{\circ} 32.7^{\prime}$ & -0.30 & - & -0.23 \\
\hline Memanbetsu & MEM & $43^{\circ} 54.4^{\prime}$ & $144^{\circ} 11.6^{\prime}$ & - & - & - \\
\hline
\end{tabular}

netic Observatory) to establish daily mean simple differences. As the whole-day mean differences were less dispersive than the night-time ones $(00 \mathrm{~h} 00 \mathrm{~m}-05 \mathrm{~h} 59 \mathrm{~m} \mathrm{L.T.})$, we adopted here the whole-day mean differences.

The whole-day daily mean differences are plotted against time as shown in Fig. 4(a). Exclusive of oscillatory changes with annual period (the amplitude less than $2 \mathrm{nT}$ ), we notice a large increasing trend of the secular change during about 3 years at AKS and NMR situated at the coastal region of the Pacific Ocean. In contrast to results from these stations, the inland stations such as SHI show low-rate secular change. Linear least-squares regression analysis on the data yielded the secular changes of $0.87 \mathrm{nT} /$ year, $1.56 \mathrm{nT} / \mathrm{year}$ and $-0.14 /$ year at AKS, NMR and SHI, respectively. Such characteristic trend at AKS and SHI has lasted at least in the past about 30 years as shown in Fig. 4(b). The secular change at each station is plotted against the horizontal distance from AKS to MMB in Fig. 5. For the sake of comparison, the data at NMR, ATK and MID are also projected onto this measurement line. It is characteristic that the secular change decreased abruptly to $-0.06 \mathrm{nT} /$ year at KKM situated at only about $15 \mathrm{~km}$ apart from AKS. The secular changes at other inland stations are also small (Fig. 5 and Table 1).

There are several possible explanations for the origin of the large secular changes: ionospheric origin, earth's core origin, ocean dynamo effect, thermal effect including heat- triggered volcanomagnetic effect, electrokinetic effect, orientation effect which will be interpreted later, and piezomagnetic effect. Among these possibilities for the source mechanism, local distribution of the large secular changes at AKS, ATK and NMR is not accounted for in terms of electric currents in the ionosphere or the earth's core. The effect of the ocean dynamo cannot explain the monotonous increase of the magnetic field at AKS for about 30 years. As AKS, ATK and NMR are situated at forearc side apart from the volcanic front as shown in Fig. 1, the volcanomagnetic effect is not a supporting factor. Other geothermal activities have not been reported in the study area. Groundwater flow can generate the magnetic field due to the electrokinetic effect (e.g., Mizutani and Ishido, 1976; Fitterman, 1981). However, AKS, ATK and NMR are situated on the impermeable layer of the Cretaceous period that prevents the groundwater flow and aquifers scarcely developed in this region (Akita, personal communication). Therefore, the electrokinetic effect may be ruled out as the origin of the large secular changes.

The difference of the geomagnetic inclination and/or declination between the reference station and the other station yields apparent secular changes at the other station when the time-dependent fields are added to the permanent field because the vectorical sum of the permanent field and the additional fields becomes different even the additional fields are the same at two stations (Tanaka et al., 1978; Davis and 


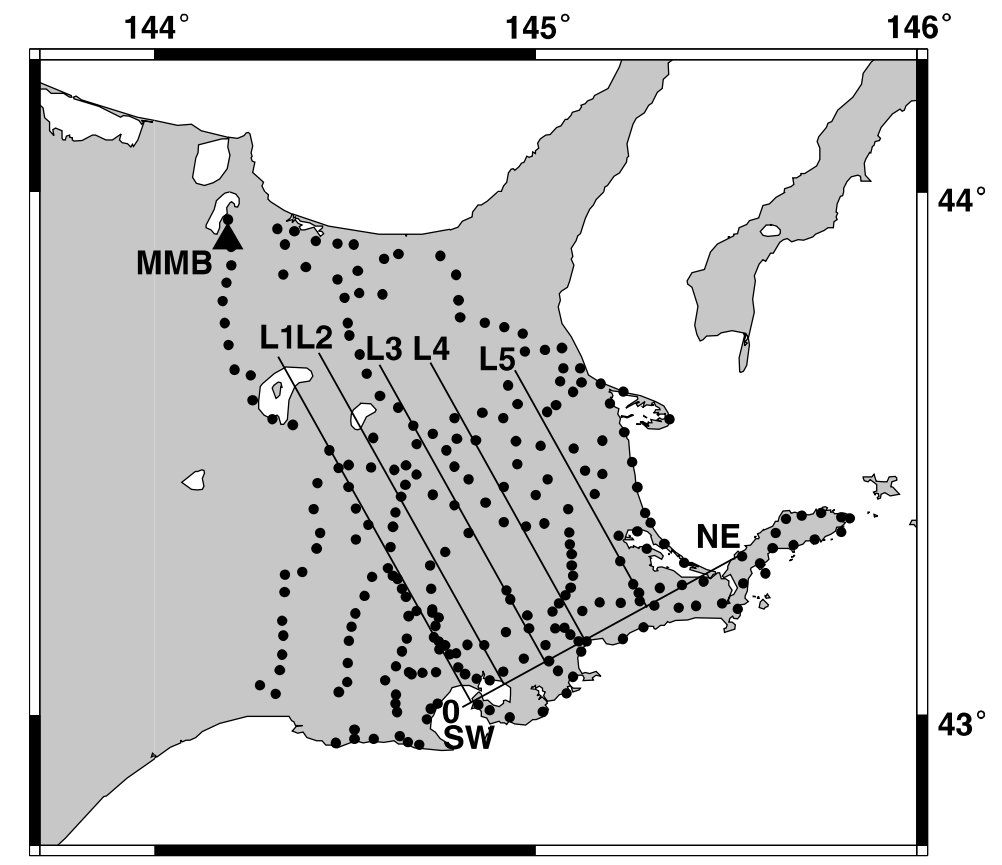

Fig. 6. Geomagnetic survey stations are shown by solid circles (after Sugisaki et al., 2001; Nishida and Watanabe, 2002). Five profile lines (L1-L5) are also shown.

Johnston, 1983). This effect is called the orientation effect by Davis and Johnston (1983). Therefore, the observed secular changes should be corrected of the orientation effect to extract the tectonomagnetic signals. Difference of the total force intensity $\Delta F$ between the reference station and the other station can be expressed as the linear relation to the additional fields through the transfer functions $a, b$ and $c$ as:

$$
\Delta F=F_{o b s}-F_{r e f}=a \Delta X+b \Delta Y+c \Delta Z,
$$

where

$$
\begin{gathered}
a=\cos I_{o b s} \cos D_{o b s}-\cos I_{r e f} \sin D_{r e f}, \\
b=\cos I_{o b s} \sin D_{o b s}-\cos I_{r e f} \sin D_{r e f}, \\
c=\sin I_{o b s}-\sin I_{r e f} .
\end{gathered}
$$

$\Delta X, \Delta Y$ and $\Delta Z$ represent the northward, eastward and downward components of the additional field, respectively. $D$ and $I$ show the declination and the inclination of the permanent field at the reference station and the other station, which are discriminated by subscripts "ref" and "obs" in Eq. (2), respectively. Then, we estimate $\Delta F$ between the reference station MMB $\left(F_{\text {ref }}\right)$ and other stations $\left(F_{\text {obs }}\right)$ to correct the orientation effect. We employed the values established by the second order geomagnetic observations by Geographical Survey Institute of Japan (2003) as $D$ and $I$ at each station. We also employed $\Delta X, \Delta Y$, and $\Delta Z$ of -12.1 nT/year, -7.4 nT/year and +19.9 nT/year, respectively, as the mean secular changes in the last 30 years in the study area (Data Analysis Center for Geomagnetism and Space Magnetism, 2003), which were independent from the local tectonomagnetic secular changes. The corrected data reveal that the large secular changes still remain at AKS, ATK and NMR as shown in Table 1 and Fig. 5.
Considering the above-mentioned examinations, we conclude that the corrected secular changes at AKS, ATK and NMR are mainly caused by piezomagnetic field changes. Piezomagnetic field change results from stress induced deviation of the magnetization of the crustal rocks and is expressed by the product of the magnetization of rocks, applied stress and stress sensitivity as shown in Section 4 of this paper (e.g., Stacey, 1964; Nagata, 1970; Sasai, 1980).

In Section 3, we will describe the results of geomagnetic mapping and model calculation of the magnetic structure in the study area to elucidate the distribution of the magnetization of rocks. Succeedingly, we will calculate the piezomagnetic field changes based on the model magnetic structure (Section 4).

\section{Geomagnetic Mapping and Modeling \\ 3.1 Geomagnetic mapping}

Although an airborne survey carried out by Geological Survey of Japan (1979) has well mapped the geomagnetic total force intensity in whole Hokkaido, the eastern part of the present study area is poorly understood. To provide more detail, we established 244 land stations (Fig. 6) using a portable proton precession magnetometer in 2000 and 2001 (Sugisaki et al., 2001; Nishida and Watanabe, 2002). Corrections for geomagnetic diurnal variations and other disturbances are made on the basis of continuous magnetic records at Memanbetsu Magnetic Observatory (MMB). The geomagnetic anomalies relative to the International Geomagnetic Reference Field (IGRF) 2000 model (Mandea and Macmillan, 2000) are shown in Fig. 7 with a contour interval of $300 \mathrm{nT}$. We can recognize the characteristic features of the anomalies as follows: positive anomalies up to a maximum of about $1,700 \mathrm{nT}$ are distributed along the coast of the Pacific Ocean, while the negative anomalies (to about $-1,650 \mathrm{nT})$ are distributed in the north of the posi- 


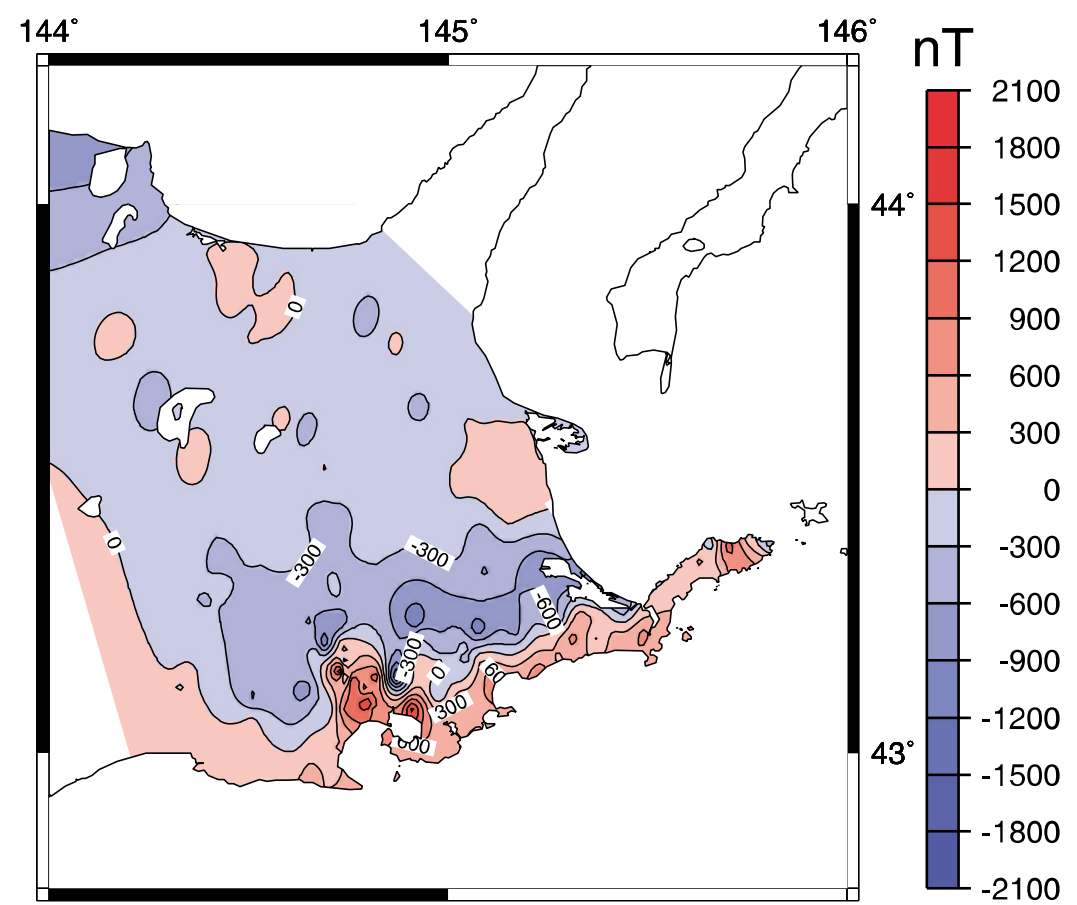

Fig. 7. Distribution of the geomagnetic anomalies relative to the IGRF 2000 values with contour interval of 300 nT. Red color represents the positive anomaly, while blue color denotes the negative anomaly (Nishida and Watanabe, 2002).

tive anomaly region. It is likely that highly and normally magnetized rocks are distributed beneath the coastal region with the NE-SW trend. In contrast, geomagnetic anomalies are relatively small in the central and northern parts of the study area where thick Quarternary sediments characterized by weak magnetization cover the surface (about 5 $\mathrm{km}$ in maximum thickness; Satoh et al., 2001).

\subsection{Modeling of the geomagnetic anomalies}

We searched for detailed magnetic structure by focusing on the geomagnetic anomalies along the coastal region of the Pacific Ocean. The observed anomalies were modeled by forward calculation based on the method of Bhattacharyya (1964). The magnetic structures producing the magnetic anomalies were approximated by a series of 3$D$ rectangular blocks, assuming that each block has uniform and parallel magnetization with the ambient geomagnetic field (a declination of $\mathrm{N} 8^{\circ} \mathrm{W}$ and an inclination of $57^{\circ}$ ). Observed and calculated anomalies along five profiles (Fig. 6) are shown in Fig. 8 with a favorable block model. Magnetization intensity of each block was adjusted from 8.0 to $10 \mathrm{~A} / \mathrm{m}$ relative to the intensity of the surrounding crust to match anomaly amplitudes. Although the resolution of the deeper structure is poor in the model calculation, it may be reasonable that calculated depths of the bottom of the magnetized blocks are $8 \mathrm{~km}$ to $16 \mathrm{~km}$ because the depth to the Curie temperature is estimated as about $10 \mathrm{~km}$ in this region (Okubo, 1999). The calculated models are somewhat complicated and are by no means unique, but the existence of highly magnetized bodies is probable from shallow depth to about $10 \mathrm{~km}$.

A series of dolerite outcrops in this region (Geological Survey of Japan, 1992) show an exceedingly high magnetization of about $10 \mathrm{~A} / \mathrm{m}$ (Fujiwara and Ohtake, 1975). However, they are not enough to explain the whole geomag- netic anomalies because the thickness of the dolerite outcrops is several tens meter or so (Yagi, 1969). In addition to these dolerites, we need thick layers of strongly magnetized rocks, such as basaltic rocks, at depths. In general, serpentinite is also a candidate to generate high magnetic anomalies because crystallized magnetite often co-exists with serpentine. However, the assumption of the existence of the serpentinite at depths may be somewhat disadvantageous: though the serpentinite often shows low electrical resistivity due to the formation of magnetite network within the serpentinite, a magnetotelluric study revealed that the crust is highly resistive (about 10,000 $\Omega \mathrm{m}$ ) in this region (Satoh et al., 2001). Future advance of detailed tectonic study is expected to identify the material causing the magnetic anomalies in this region.

\section{Calculation of Piezomagnetic Field}

From experimental and theoretical studies, the relationship between uniaxial mechanical stress, $\sigma$, and the associated changes in rock magnetization, $\Delta J$, is expressed as follows (Stacey, 1964; Nagata, 1970; Stacey and Johnston, 1972):

$$
\begin{array}{r}
\Delta \boldsymbol{J}^{\|}=\sigma\left(\beta_{r} \boldsymbol{J}_{0}^{\|}+\beta_{i} \chi_{0} \boldsymbol{H}^{\|}\right) \\
\Delta \boldsymbol{J}^{\perp}=-\frac{\sigma}{2}\left(\beta_{r} \boldsymbol{J}_{0}^{\perp}+\beta_{r} \chi_{0} \boldsymbol{H}^{\perp}\right)
\end{array}
$$

in which the superscripts $\|$ and $\perp$ represent the magnetization components parallel and perpendicular to the applied stress direction, respectively. $\boldsymbol{J}_{0}$ is the remanent magnetization of the rock and $\boldsymbol{H}$ is the ambient geomagnetic field. $\chi_{0}$ denotes the initial magnetic susceptibility of the rock. Proportional coefficients $\beta_{r}$ and $\beta_{i}$ are called the stress sensitivity of remanence and the stress sensitivity of susceptibility, respectively. 

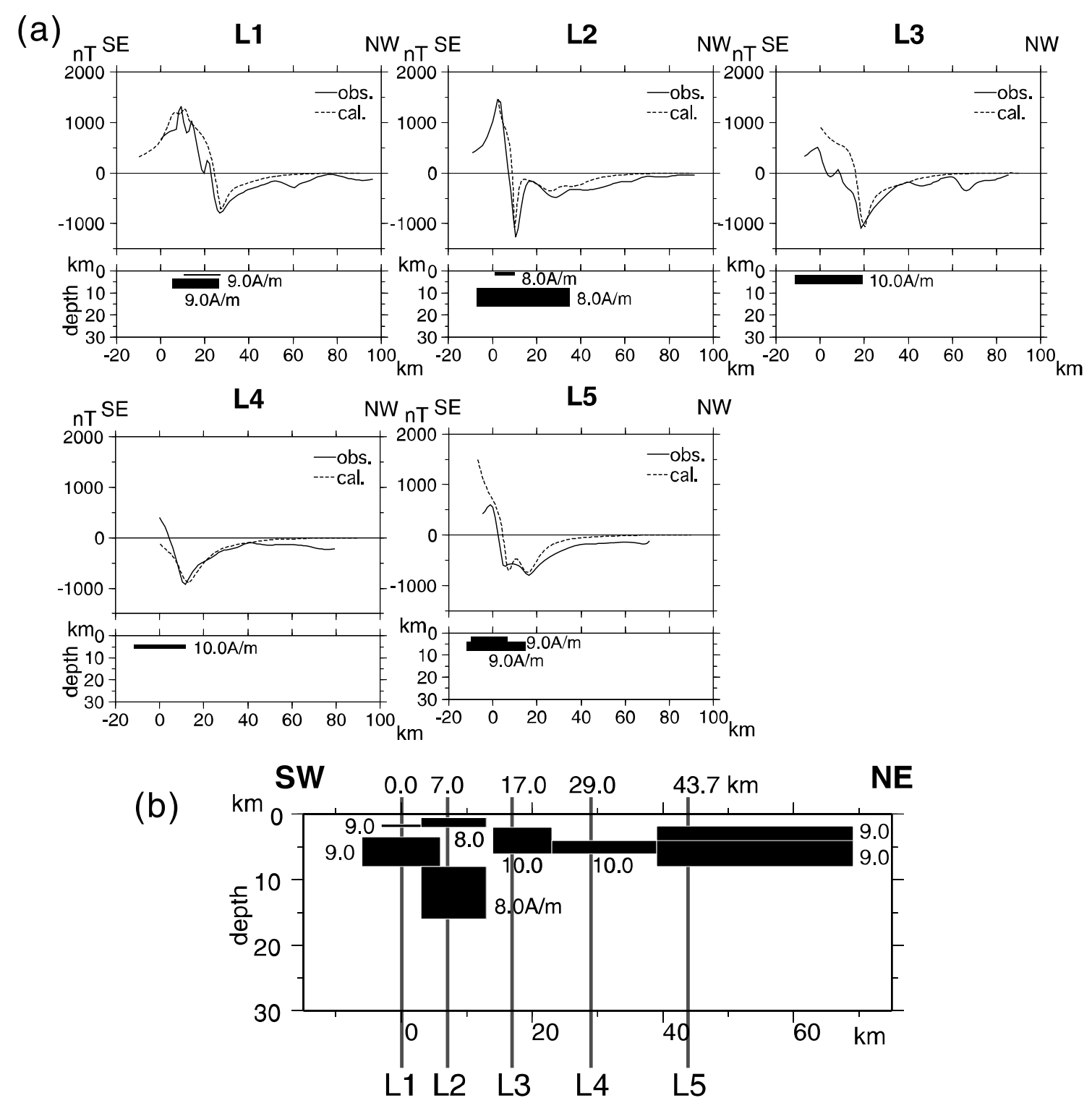

Fig. 8. (a) Observed (solid curves) and calculated (dashed curves) geomagnetic anomalies along the profiles shown in Fig. 6. Model cross sections are also shown. (b) Model cross section along the SW-NE line (modified from Takahashi, 2002). Values of magnetization (unit: A/m) are also indicated.

The piezomagnetic potential $\Delta W$ produced by the change of the magnetization $(\Delta J)$ can be written by the following volumetric integral over the magnetized region $V$ :

$$
\Delta W=-\iiint_{V} \Delta \boldsymbol{J} \cdot \frac{\boldsymbol{r}}{R^{3}} d V,
$$

where $R$ denotes the distance between the observation point and the arbitrary point within the medium. $r$ shows the position vector. The piezomagnetic field change, $\Delta \boldsymbol{M}$, is expressed by derivation of the piezomagnetic potential of Eq. (4):

$$
\Delta \boldsymbol{M}=-\frac{\partial \Delta W}{\partial \boldsymbol{r}}
$$

When we assume a uniform stress distribution, $\Delta \boldsymbol{J}$ of magnetized region becomes constant. Therefore, the volumetric integral of Eq. (4) becomes as follows:

$$
\Delta W=-\Delta \boldsymbol{J} \iiint_{V} \frac{\boldsymbol{r}}{R^{3}} d V .
$$

As this equation is identical with that of the magnetic anomalies produced by a uniformly magnetized region, we can easily calculate by using the closed-form solution provided by, for example, Bhattacharyya (1964).

When we assume many magnetized blocks, the geomagnetic field change $(\Delta \boldsymbol{M})$ at a certain point on the earth's surface would be approximated by the sum of the piezomagnetic field derived from each block. Then, we perform the piezomagnetic modeling under the condition that uniform regional tectonic stress has been applied horizontally on highly magnetized rectangular blocks buried within the non-magnetized half-space medium. In general, it is difficult to evaluate the in-situ stress field, especially for the vertical component. However, the predominance of the horizontal stress field may be good assumption in the subduction zone because many reverse fault type earthquakes occur. In order to obtain $\Delta \boldsymbol{J}$ in Eq. (3), the stress field $(\sigma)$ is estimated from the observed strain rate $\left(4 \times 10^{-7} /\right.$ year $)$ with principal axis of NW-SE direction (mean direction: 


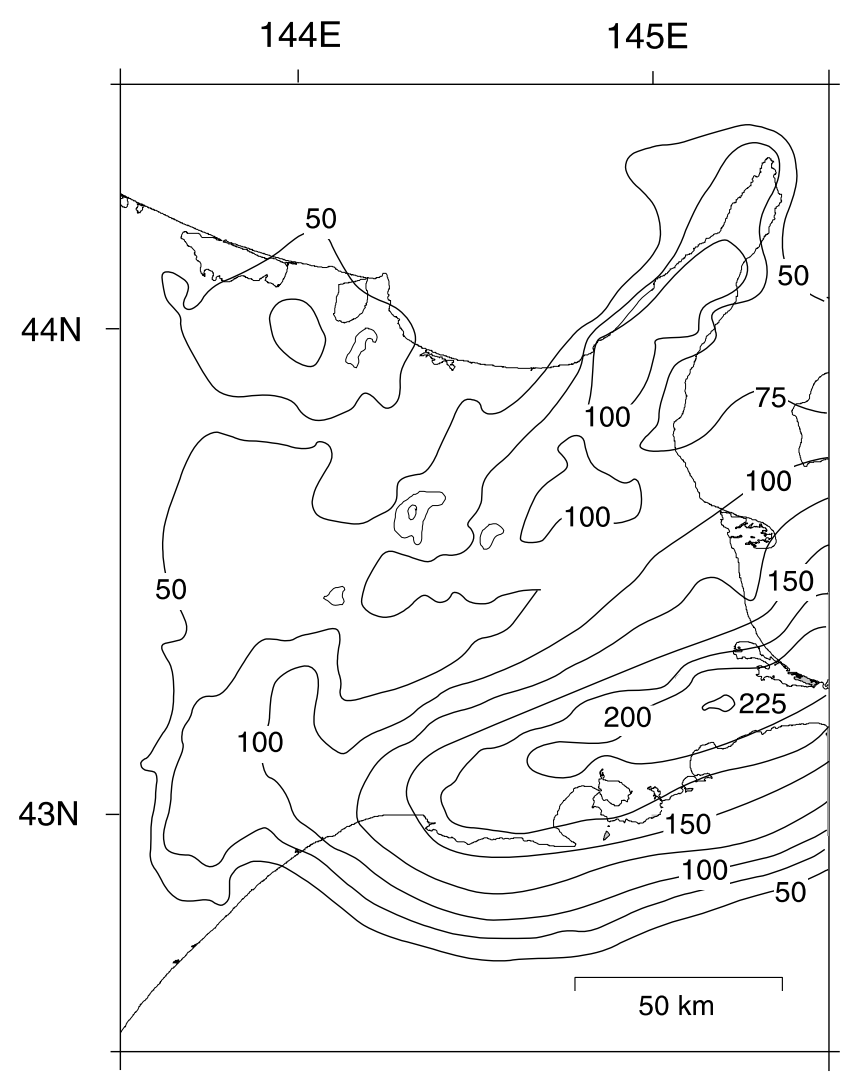

Fig. 9. Distribution of the Bouguer anomaly in the study area (modified from Yamamoto and Moriya, 1989). Contour interval is $25 \mathrm{mgal}$.

$\mathrm{N} 48^{\circ} \mathrm{W}-\mathrm{S} 52^{\circ} \mathrm{E}$ ) (Ishikawa and Hashimoto, 1999), assuming a homogeneous and isotropic elastic medium with the rigidity of $3.5 \times 10^{4} \mathrm{MPa}$ and the Poisson ratio of 0.25 . The subsurface magnetic structure described in Section 3 is used as the magnetization of the rocks. A value of $2 \times 10^{-3}$ $\mathrm{MPa}^{-1}$ is used for both the stress sensitivities $\beta_{r}$ and $\beta_{i}$ as ordinarily assumed one (Stacey and Johnston, 1972). The initial magnetic susceptibility $\chi_{0}$ is adopted as $4 \pi \times 10^{-2}$ which is rather large value for igneous rocks.

The calculation of the piezomagnetic field is made on the earth's surface of the study area and the values along the L1 line (Fig. 6) are compared with the secular changes corrected for the orientation effect as shown in Fig. 5. Calculated abrupt enhancement of the amplitude follows well the corrected secular changes, although the calculated maximum amplitude appears at the slightly different place from AKS (difference: nearly $10 \mathrm{~km}$ ). Such enhancement is explained by the fact that magnetic fields arising from stressinduced magnetic dipoles tend to cancel with one another within a uniformly magnetized block, while they do not at the boundary between the magnetized and non-magnetized mediums (Oshiman, 1990; Oshiman et al., 1991b; Utsugi, 1999, 2000). The calculated maximum amplitude, however, explains only about $15 \%$ of the observed value at AKS despite the use of highly magnetized medium of $8-10 \mathrm{~A} / \mathrm{m}$.

\section{Discussion}

Many efforts have been made to observe geomagnetic changes associated with earthquakes, long-term crustal deformations, filling of reservoirs and so on. In some cases, the observed piezomagnetic amplitudes were successfully explained by assuming a seismic fault model, magnetization of the crust (an order of $1 \mathrm{~A} / \mathrm{m}$ ) and stress sensitivity (an order of $10^{-3} \mathrm{MPa}^{-1}$ ) as in examples of the Landers earthquake (Johnston et al., 1994), a Parkfield fault model (Stuart et al., 1995: long-term crustal deformation) and the 1978 Higashi-Izu earthquake (Sasai and Ishikawa, 1997: pre- and co-seismic). However, the calculated piezomagnetic changes were often underestimated in comparison with the observed ones in the cases of reservoirs loading when the stress sensitivity and magnetization of the crust were assumed to be of the orders of $10^{-3} \mathrm{MPa}^{-1}$ and $1 \mathrm{~A} / \mathrm{m}$, respectively. We had to assume the magnetization larger than $5 \mathrm{~A} / \mathrm{m}$ for interpretating the observed signals (Zhan, 1989). Here, we quote from Sasai (1991b): "Then, a question arises: Are all the reservoirs in the world located on an intense magnetization of $5 \mathrm{~A} / \mathrm{m}$ or much more?". However, the magnetization of $5 \mathrm{~A} / \mathrm{m}$ or much more might be too large in the Nichinan reservoir region because the geology of this region is composed of negligibly magnetized granite (Oshiman et al., 1991a).

In some cases of the previous field studies, both magnetization and stress sensitivity of the crust were no more than assumption and were treated as parameters to be adjusted for interpreting the amplitudes of the observed piezomagnetic signals. We modeled the detailed magnetic structure as mentioned above and used the tectonic stress deduced from the observed strain rate in the study area to make more realistic comparison between the observed and calculated piezomagnetic signals. However, we failed to reproduce the amplitude of the secular changes at AKS when we adopted the stress sensitivity of ordinarily assumed values $\left(\beta_{r}=\beta_{i}=2 \times 10^{-3} \mathrm{MPa}^{-1}\right)$. We may need the stress sensitivity of remanence and/or susceptibility of the order of $10^{-2} \mathrm{MPa}^{-1}$ for the quantitative interpretation.

As previously described, Hamano (1983) found that the stress sensitivity increased with porosity of rocks due to stress intensification multiplying local stress within the sample. However, it is hard to think that the highly magnetized rocks in the present study area are porous because the distribution of the high gravity anomalies up to a maximum of 227 mgal (Fig. 9) (Yamamoto and Moriya, 1989) correlates well with that of the remarkable geomagnetic anomalies along the coastal region of the Pacific Ocean, suggesting that the material causing both the geomagnetic and gravity anomalies is common and dense.

Summing up the above-mentioned discussions, we conclude inevitably that the large secular changes at AKS, ATK and NMR result from a larger stress sensitivity than ordinarily assumed. In addition to the effect of porosity of rocks (Hamano, 1983), some possible mechanisms of the enhancement of the stress sensitivity have been proposed. As Stacey and Johnston (1972) calculated, stress sensitivity increases with titanium content in titanomagnetites. An experimental study by Kean et al. (1976) revealed that the stress sensitivity tends to be large as magnetic grain size becomes large. Kinoshita and Hamano (1980) pointed out that the stress sensitivity might depend on the size of rock samples in laboratory experiments, although detailed experiments are few. In any case, however, it is presently difficult 
to evidence the detailed mechanism of enhancement of the stress sensitivity in the study area.

\section{Conclusion}

In order to provide quantitative interpretation of the large geomagnetic secular changes in the eastern part of Hokkaido, NE Japan, we developed a piezomagnetic model in which the regional tectonic stress (deduced from the observed strain rate, $4 \times 10^{-7} /$ year) is applied to highly magnetized rocks $(8-10 \mathrm{~A} / \mathrm{m})$ deduced from modeling of the observed geomagnetic anomalies. The secular changes are well explained by the piezomagnetic model when we assume the stress sensitivity of the order of $10^{-2} \mathrm{MPa}^{-1}$ which is one order larger than the ordinarily used value. The reason why the rocks in the study area have the larger stress sensitivity is left to be solved. Further studies of laboratory experiments of the piezomagnetism are required to clarify the mechanisms of the enhancement of the stress sensitivity.

Acknowledgments. Gratitude is expressed to Tokumitsu Maekawa, Hideyuki Satoh, Kazuyuki Hirano and Tomohiro Watanabe for their great efforts in the field work. We are greatly indebted to suggestions by Yoichi Sasai, Yoshikazu Tanaka, Takeshi Hashimoto and Oguz Ozel to improve this paper. Malcolm J. S. Johnston and Naoto Oshiman provided useful review comments to complete the manuscript. We are very thankful to Akkeshi Marine Station of Hokkaido University and Shibecha Experimental Forest of Kyoto University for their kind help in the course of observation.

\section{References}

Abdullabekov, K. N., Ye. Brdaliyev, A. N. Pushkov, and V. A. Shapiro, Local variations of the geomagneti field during the filling of a reservoir, Geomag. Aeron., 19, 204-206, 1979 (English translation).

Bhattacharyya, B. K., Magnetic anomalies due to prism-shaped bodies with arbitrary polarization, Geophys., 29, 517-531, 1964.

Data Analysis Center for Geomagnetism and Space Magnetism, Kyoto Univ., http://swdedb.kugi.kyoto-u.ac.jp/, 2003.

Davis, P. M. and F. D. Stacey, Geomagnetic anomalies caused by a manmade lake, Nature, 240, 348-349, 1972.

Davis, P. M. and M. J. S. Johnston, Localized geomagnetic field changes near active faults in California 1974-1980, J. Geophys. Res., 88. 94529460, 1983.

Fitterman, D. V., Correction to "Theory of electrokinetic-magnetic anomalies in a faulted half-space", J. Geophys. Res., 86, 9585-9588, 1981.

Fujiwara, Y. and T. Ohtake, Paleomagnetism of late Cretaceous alkaline rocks in the Nemuro peninsula, Hokkaido, N. E. Japan, J. Geomag. Geoelectr., 26, 549-558, 1975.

Geographycal Survey Institute of Japan, http://vldb.gsi.go.jp/sokuchi/ geomag/, 2003.

Geological Survey of Japan, 1:200,000 Total intensity aeromagnetic maps, 1979.

Geological Survey of Japan, 1:1,000,000 Geological map of Japan, Third edition, 1992.

Hamano, Y., Experiments on the stress sensitivity of natural remanent magnetization, J. Geomag. Geoelectr., 35, 155-172, 1983.

Hasegawa, K., F. Muromatsu, M. Sugawara, M. Hashimoto, K. Koike, and N. Mizuno, Observation for earthquake prediction in the eastern part of Hokkaido and the secular variation of conductivity anomalies in and around Attoko, Technical Rep. Mag. Observatory, 22, 49-63, 1983 (in Japanese).

Ishikawa, N. and M. Hashimoto, Average horizontal crustal strain rates in Japan during interseismic period deduced from geodetic surveys (Part 2), Zisin (J. Seismol. Soc. Jpn.), 52, 299-315, 1999 (in Japanese with English abstract).

Johnston, M. J. S., S. A. Silverman, R. J. Mueller, and K. S. Breckenridge, Secular variation, crustal contribution, and tectonic activity in California, 1976-1984, J. Geophys. Res., 90, 8707-8717, 1985.

Johnston, M. J. S., R. J. Mueller, and Y. Sasai, Magnetic field observations in the near-field of the 28 June 1992 Mw 7.3 Landers, California, earthquake, Bull. Seism. Soc. Amer., 84, 792-798, 1994.

Kapitsa, S. P., Magnetic properties of eruptive rocks under mechanical stresses, Izv. Akad. Nauk U.S.S.R., 86, 521-523, 1955.

Kean, W. R., R. Day, M. Fuller, and V. A. Schmidt, The effect of uniaxial Compression on the initial susceptibility of rocks as a function of grain size and composition of their constituent titanomagnetite, J. Geophys. Res., 81, 861-872, 1976.

Kinoshita, H. and Y. Hamano, Piezomagnetism, in Earthquake Prediction I: Electromagnetic approach, pp. 51-82, Gakkai Shuppan Center, Tokyo, 1980 (in Japanese).

Mandea, M. and S. Macmillan, International Geomagnetic Reference Field - the eighth generation, Earth Planets Space, 52, 1119-1124, 2000.

Mizutani, M. and T. Ishido, A new interpretation of magnetic field variation associated with the Matsushiro earthquakes, J. Geomag. Geoelectr., 28, 179-188, 1976.

Nagata, T., Basic magnetic properties of rocks under the effect of mechanical stresses, Tectonophysics, 9, 167-195, 1970.

Nagata, T. and H. Kinoshita, Effect of hydrostatic pressure on magnetostriction and magnetocrystalline anisotropy of magnetite, Phys. Earth Planet. Int., 1, 44-48, 1967.

Niida, K. and N. Kato, Cretaceous arc-trench systems in Hokkaido, Monograph Assoc. Geol. Collab., 31, 379-402, 1986 (in Japanese with English abstract).

Nishida, Y. and T. Watanabe, Geomagnetic survey in the eastern part of Hokkaido, NE Japan (2): Supplemental data report, J. Fac. Sci., Hokkaido Univ., Ser. 7, 11, 895-900, 2002.

Okubo, Y., Temperature analysis in the Earth's crust, in Handbook of Geophysical Exploration, edited by Soc. Exploration Geophys. Jpn., pp. 586-588, 1999 (in Japanese).

Oshima, H., T. Maekawa, M. Utsugi, and Y. Nishida, Repeated survey of gravity and geomagnetic total force intensity in the eastern part of Hokkaido after the Kushiro-Oki Earthquake of January 15, 1993, Geophys. Bull., Hokkaido Univ., 57, 23-33, 1994 (in Japanese with English abstract).

Oshiman, N., Enhancement of tectonomagnetic change due to non-uniform magnetization in the Earth's crust-two dimensional case studies, $J$. Geomag. Geoelectr., 42, 607-619, 1990.

Oshiman, N., Y. Sasai, J. Miyakoshi, R. Nishida, and I. Shiozaki, Continuous observation of piezomagnetic changes due to ground loading by Lake Nichinan, Tottori, Japan, Proc. Conductivity Anomaly Symp. (1991), 137-148, 1991a (in Japanese).

Oshiman, N., M. K. Tunçes, Y. Honkura, S. Baris, O. Yazici, and Ishikara, A strategy of tectonomagnetic observation for monitoring possible precursors to earthquakes in the western part of the North Anatolian Fault Zone, Turkey, Tectonophys., 193, 359-368, 1991b.

Sasai, Y., Application of the elasticity theory of dislocations to tectonomagnetic modelling, Bull. Earthq. Res. Inst., Univ. Tokyo, 55, 387-447, 1980.

Sasai, Y., Tectonomagnetic modeling on the basis of the linear piezomagnetic effect, Bull. Earthq. Res. Inst., Univ. Tokyo, 66, 587-722, 1991a.

Sasai, Y., Tectonomagnetic modeling on the basis of the linear piezomagnetic effect, Ph.D. Thesis, Univ. Tokyo, Tokyo, pp. 164, 1991b.

Sasai, Y., Piezomagnetic fields produced by dislocation sources, Surv. Geophys., 15, 363-382, 1994.

Sasai, Y., Tectonomagnetic modeling based on the piezomagnetism: A review, Annali di Geofisica, 44(2), 361-368, 2001.

Sasai, Y. and Y. Ishikawa, Changes in the geomagnetic total force intensity associated with the anomalous crustal activity in the eastern part of the Izu Peninsula (3) — The east off Izu Peninsula earthquake of 1980-, Bull. Earthq. Res. Inst., Univ. Tokyo, 55, 1101-1113, 1980 (in Japanese with English abstract).

Sasai, Y. and Y. Ishikawa, Seismomagnetic models for earthquakes in the eastern part of Izu Peninsula, Central Japan, Annali di Geofisica, 40(2), 463-478, 1997.

Satoh, H., Y. Nishida, Y. Ogawa, M. Takada, and M. Uyeshima, Crust and upper mantle resitivity structure in the southwestern end of the Kuril Arc as revealed by the joint analysis of conventional MT and network MT data, Earth Planets Space, 53, 829-842, 2001.

Stacey, F. D., The seismomagnetic effect, Pageoph, 58, 5-22, 1964.

Stacey, F. D. and M. J. S. Johnston, Theory of the piezomagnetic effect in titanomagnetite-bearing rocks, Pageoph, 97, 146-155, 1972.

Stuart, W. D., P. O. Banks, Y. Sasai, and S-W. Liu, Piezomagnetic field for Parkfield fault model, J. Geophys. Res., 100, 24101-24110, 1995.

Sugisaki, Y., K. Takahashi, and Y. Nishida, Geomagnetic survey in the eastern part of Hokkaido, NE Japan: A data report, J. Fac. Sci., 
Hokkaido Univ., Ser. 7, 11, 811-820, 2001.

Takahashi, K., Magnetic anomalies in the eastern part of Hokkaido and a crustal magnetic model, B. Sci Thesis, Hokkaido Univ., Sapporo, pp. 28, 2002.

Tanaka, Y., H. Masuda, H. Kawamura, K. Ohchi, Y. Kato, H. Baba, and T. Yoshino, Geomagnetic survey of total force intensity on Aso volcanic region-1977, Rep. Joint Geophys, Geochem. Obs. of Aso Volcano, 3139, 1978 (in Japanese).

Utsugi, M., A theoretical study on seismomagnetic effect considering the inhomogeneously magnetized Earth's crust, Ph.D. Thesis, Hokkaido Univ. Sapporo, pp. 128, 1999.

Utsugi, M., A computer program for the calculation of piezomagnetic field due to a spherical pressure source (Mogi model) in the inhomogeneously magnetized crust, J. Fac. Sci., Hokkaido Univ., Ser. 7, 11, 739-751, 2000.

Utsugi, M., Y. Nishida, and Y. Sasai, Piezomagnetic potentials due to an inclined rectangular fault in a semi-infinite medium, Geophys. J. Int.,
140, 479-492, 2000.

Yagi, K., Petrology of the alkaline dolerites of the Nemuro peninsula, Japan, Geol. Soc. Amer. Mem., 115, 103-149, 1969.

Yamamoto, A. and T. Moriya, Gravity anomalies and structure of Hokkaido, Chikyu Monthly, 11, 377-385, 1989 (in Japanese).

Yukutake, T. and H. Tachinaka, Geomagnetic variation associated with stress change within a semi-infinite elastic earth caused by a cylindrical force source, Bull. Earthq. Res. Inst., Univ. Tokyo, 45, 785-798, 1967.

Zhan, Z., Investigations of tectonomagnetic phenomena in China, Phys. Earth Planet. Interiors, 57, 11-22, 1989.

Zlotnicki, J. and F. H. Cornet, A numerical model of earthquake-induced piezomagnetic anomalies, J. Geophys. Res., 91, 709-718, 1986.

Y. Nishida (e-mail: nishida@ares.sci.hokudai.ac.jp), Y. Sugisaki, K Takahashi, M. Utsugi, and H. Oshima 